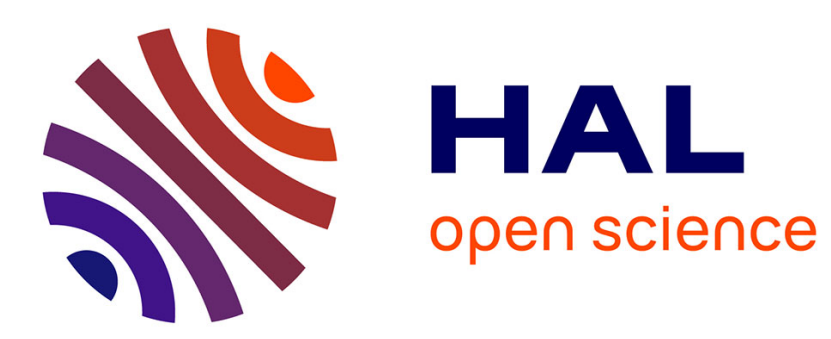

\title{
Singularity Analysis of Lower-Mobility Parallel Robots with an Articulated Nacelle
}

Semaan Amine, Daniel Kanaan, Stéphane Caro, Philippe Wenger

\section{To cite this version:}

Semaan Amine, Daniel Kanaan, Stéphane Caro, Philippe Wenger. Singularity Analysis of LowerMobility Parallel Robots with an Articulated Nacelle. Advances in Robot Kinematics, Jun 2010, Piran-Portoroz, Slovenia. pp.273-282. hal-00642259

\section{HAL Id: hal-00642259 https://hal.science/hal-00642259}

Submitted on 17 Nov 2011

HAL is a multi-disciplinary open access archive for the deposit and dissemination of scientific research documents, whether they are published or not. The documents may come from teaching and research institutions in France or abroad, or from public or private research centers.
L'archive ouverte pluridisciplinaire HAL, est destinée au dépôt et à la diffusion de documents scientifiques de niveau recherche, publiés ou non, émanant des établissements d'enseignement et de recherche français ou étrangers, des laboratoires publics ou privés. 


\title{
Singularity Analysis of Lower-Mobility Parallel Robots with an Articulated Nacelle
}

\author{
Semaan Amine, Daniel Kanaan, Stéphane Caro and Philippe Wenger \\ Institut de Recherche en Communications et Cybernétique de Nantes, France, \\ e-mail: \{firstname.lastname $\} @ i r c c y n . e c-n a n t e s . f r$
}

\begin{abstract}
This paper presents a generic approach to analyze the singularity of robots with an articulated nacelle like the $H 4$ robot. Using screw theory, the concept of equivalent twist graph is introduced in order to characterize the constraint wrenches and the actuation wrenches applied to the moving platform. Using Grassmann-Cayley Algebra, the geometric conditions associated with the dependency of six Plücker vectors of finite and infinite lines in the projective space $\mathbb{P}_{3}$ are reformulated in the superbracket decomposition in order to characterize geometrically the parallel singularities of the robot.

Key words: Grassmann-Cayley Algebra, superbracket, screw theory, parallel manipulators, singu-
\end{abstract} larities, twist graph, nacelle.

\section{Introduction}

Parallel singularities are critical configurations in which a parallel manipulator loses its stiffness and gains one or more degrees of freedom (DOF). They can be found using either numerical, analytical or geometrical methods. For Gough Stewart parallel manipulators, the rows of the inverse Jacobian matrix are Plücker coordinate vectors of six finite lines that are six actuation forces applied by the actuators to the moving platform. The parallel singularities of such manipulators that Merlet [1] analyzed using Grassmann line geometry occur when those lines become linearly dependent. Ben-Horin and Shoham analyzed the parallel singularities of 6-DOF parallel manipulators using Grassmann-Cayley Algebra (GCA) [2, 3]. Contrary to 6-DOF parallel manipulators, the legs of a lower-mobility parallel manipulator have a connectivity smaller than six and, in turn, each leg constrains partly the motion of the moving platform. When the legs lose their ability to constrain the moving platform, a socalled constraint singularity occurs [4]. Kanaan et al. [5] enlarged the application of GCA to lower-mobility manipulators, in which the rows of the $6 \times 6$ inverse Jacobian are not necessarily finite lines (zero pitch wrenches) but can be also infinite lines (infinite pitch wrenches). These wrenches, also known as governing lines, are actuation and constraint wrenches applied to the moving platform. Choi et al. [6] 
analyzed the singularities of the $H 4$ robot using screw theory, while Wu et al. [7] analyzed them using line geometry.

In this paper, we present a methodology for the singularity analysis of robots with an articulated nacelle. This methodology is general and applied to the $H 4$ robot. To deal with robots with complex architectures like the $H 4$ robot, we introduce the concept of twist graph. Each leg of this robot contains a closed loop (the 4S parallel linkage) and can be replaced by a virtual serial chain having the same twist system as the leg in the equivalent twist graph of the robot. The twist graph of the $H 4$ robot is obtained in order to characterize the constraint and actuation wrenches applied to its end effector. Then, the parallel singularity conditions of the robot are derived using its wrench diagram in the projective space $\mathbb{P}_{3}$ and the superbracket decomposition. Finally, some singularity configurations are illustrated geometrically.

\section{Grassmann-Cayley Algebra in the Projective Space $\mathbb{P}_{3}$}

The Grassmann-Cayley Algebra (GCA), also known as exterior algebra, was developed by $\mathrm{H}$. Grassmann as a calculus for linear varieties operating on extensors with the operators join and meet associated with the union and intersection of vector spaces of extensors. The bracket is a determinant that satisfies special product relations called sygzygies. In the projective space $\mathbb{P}_{3}$, extensors could represent entities such as points, lines or planes. Points are represented with their homogeneous coordinates while lines and planes are represented with their Plücker coordinates. The notion of extensor makes it possible to work at the symbolic level, and therefore, to produce coordinate-free algebraic expressions for the geometric singularity conditions of spatial parallel manipulators. For further details on GCA the reader is referred to $[2,8]$.

\section{Theory of Reciprocal Screws}

Screw theory is suitable for the type synthesis and the study of the instantaneous motion of parallel manipulators. The principle of reciprocal screws was studied in $[9,10,11]$ and then developed in $[12,13,14,15]$. The method deals with the reciprocity condition between two screw systems to characterize for each leg-chain, the constraint wrench system reciprocal to its twist system as well as the actuation wrench system obtained by locking actuators. In a non-singular configuration, constraint wrenches and actuation wrenches form a 6-system.

A twist is a screw representing the instantaneous motion of a rigid body, a wrench is a screw representing a system of forces and moments acting on a rigid body. Let $\varepsilon$ denotes a twist and $\tau$ denotes a wrench. An infinite pitch twist $\varepsilon_{\infty}$ represents a pure translation of a prismatic joint $P$ and a zero pitch twist $\varepsilon_{0}$ represents a pure rotation of a revolute joint $R$. A pure force constrains the translation along its line direction 
and is represented by a zero pitch wrench $\tau_{0}$. A pure moment constrains the rotation about its direction and is represented by an infinite pitch wrench $\tau_{\infty}$. A zero pitch screw represents the Plücker coordinate vector of a finite line in $\mathbb{P}_{3}$. An infinite pitch screw represents the Plücker coordinate vector of an infinite line in $\mathbb{P}_{3}$.

A screw system of order $n(0 \leq n \leq 6)$, also called $n$-system, comprises all the screws that are linearly dependent on $n$ given linearly independent screws. Any set of linearly independent screws within a $n$-system forms a basis of this system. A $n$ screw system can be replaced by another equivalent $n$-system by applying a linear transformation to the basis of the first one [16]. The twist system $\mathrm{T}^{i}$ and the wrench system $\mathrm{W}^{i}$ of a serial kinematic chain composed of $f$ joints are given by:

$$
\mathrm{T}^{i}=\sum_{j=1}^{f} \mathrm{~T}_{j}, \quad \mathrm{~W}^{i}=\bigcap_{j=1}^{f} \mathrm{~W}_{j}
$$

The twist system $\mathrm{T}$ and the wrench system $\mathrm{W}$ of a parallel kinematic chain composed of $m$ serial chains are given by:

$$
\mathrm{T}=\bigcap_{i=1}^{m} \mathrm{~T}^{i}, \quad \mathrm{~W}=\sum_{i=1}^{m} \mathrm{~W}^{i}
$$

The twist system $\mathrm{T}$ and the wrench system $\mathrm{W}$ of a given kinematic chain are reciprocal to each other: $\mathrm{T}=\mathrm{W}^{\perp}$ and $\mathrm{W}=\mathrm{T}^{\perp}$, the operator $\perp$ denoting the reciprocity of two screw systems. If $t$ denotes the twist system order, then the wrench system order is $w=6-t$. Any twist in $\mathrm{T}$ is reciprocal to any wrench in $\mathrm{W}$ and vice versa [15]. Two screws are reciprocal to each other if their orthogonal product is equal to zero. Two zero pitch screws are reciprocal to each other if and only if their axes are coplanar. A zero pitch screw is reciprocal to an infinite pitch screw if their directions are orthogonal to each other. Two infinite pitch screws are always reciprocal to each other.

\section{The $H 4$ Constraint Analysis}

The $H 4$ robot shown in Fig. 1 belongs to a new family of 4-DOF parallel robots designed for high-speed pick and place operations [17, 18]. A kinematic graph of the $H 4$ robot was given in [19]. The $H 4$ robot is composed of four identical legs $l_{i}=\underline{\mathrm{R}}^{i}-(4 \mathrm{~S})^{i},(i=1, \ldots, 4)$, attached to a common base $(B)$ and linked to the end effector $(E)$ by means of an articulated nacelle. The nacelle is composed of three bodies: (i) $b_{I}$, connecting $l_{1}$ and $l_{2}$ in parallel and giving a resulting chain $l_{12}$; (ii) $b_{I I}$ connecting $l_{3}$ and $l_{4}$ in parallel and giving a resulting chain $l_{34}$; (iii) and $(E)$, linked to $b_{I}$ and $b_{I I}$ with two revolute joints $\mathrm{R}_{I}$ and $\mathrm{R}_{I I}$ respectively, and carrying the end effector of the robot. Finally, the $H 4$ robot has two compound legs: $L_{I}=l_{12}-\mathrm{R}_{I}$ and $L_{I I}=l_{34}-\mathrm{R}_{I I}$. 
(B)

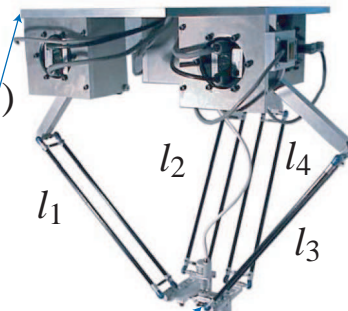

(E)

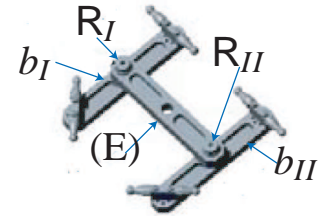

Fig. 1 The $H 4$ robot

\subsection{Twist System and Wrench System of the 4S Parallel Linkage}

A parallel linkage (4S) ${ }^{i}$ of $i$ th $l_{i}$ is represented in Fig. 2a and its kinematic graph is shown in Fig. 2b. In this graph, a segment denotes a kinematic joint and a circle denotes a link. The $(4 S)^{i}$ linkage is a parallel kinematic chain composed of two serial chains $S_{m}^{i}-S_{p}^{i}$ and $S_{n}^{i}-S_{q}^{i}$, where $S_{m}^{i}, S_{n}^{i}, S_{p}^{i}$ and $S_{q}^{i}$ are four spherical joints centered at points $m, n, p$ and $q$ respectively. In a general configuration, a $4 S$ linkage is not a parallelogram. However, the $H 4$ robot is designed in such a way that the $(4 \mathrm{~S})^{i}$ is a parallelogram in all configurations [17], i.e., $m n \| p q$ and $m p \| n q$. Note that for the $i$ th leg $l_{i}, \mathbf{r}_{p}^{i}$ denotes the position vector of point $p, \mathbf{s}_{m p}^{i}$ denotes a unit vector directed along line $m p$ and $\mathbf{n}^{i}$ denotes a unit vector normal to the plane of the parallelogram linkage.

The wrench system of $S_{m}^{i}-S_{p}^{i}$ includes all $\tau_{0}$ passing through $m$ and $p$. It is a 1system: $\mathrm{W}_{m p}=\operatorname{span}\left(\hat{\tau}_{01}\right)$ where ${ }^{1} \hat{\tau}_{01}=\left(\mathbf{s}_{m p}^{i}, \mathbf{r}_{p}^{i} \times \mathbf{s}_{m p}^{i}\right)$. Similarly, the wrench system of $\mathbf{S}_{n}^{i}-\mathbf{S}_{q}^{i}$ is: $\mathrm{W}_{n q}=\operatorname{span}\left(\hat{\tau}_{02}\right)$ where $\hat{\tau}_{02}=\left(\mathbf{s}_{n q}^{i}, \mathbf{r}_{q}^{i} \times \mathbf{s}_{n q}^{i}\right)$. From Eq.(2), the wrench system of the (4S) $)^{i}$ linkage is $\mathrm{W}_{4 \mathrm{~S}}=\mathrm{W}_{m p}+\mathrm{W}_{n q}=\operatorname{span}\left(\hat{\tau}_{01}, \hat{\tau}_{02}\right)$. Since $\mathbf{s}_{m p}^{i}$ and $\mathbf{s}_{n q}^{i}$ are parallel, $\mathrm{W}_{4 S}=\operatorname{span}\left(\hat{\tau}_{01}, \hat{\tau}_{\infty 1}\right)$, where ${ }^{2} \hat{\tau}_{\infty 1}=\hat{\tau}_{02}-\hat{\tau}_{01}=\left(\mathbf{0}, \mathbf{s}_{m p}^{i} \times \mathbf{s}_{m n}^{i}\right)[16] . \mathrm{W}_{4 S}$ is a 2-system, its twist system $\mathrm{T}_{4 \mathrm{~S}}=\mathrm{W}_{4 \mathrm{~S}}^{\perp}$ is a 4-system spanned by: $\hat{\varepsilon}_{01}^{i}=\left(\mathbf{s}_{m p}^{i}, \mathbf{r}_{p}^{i} \times\right.$ $\left.\mathbf{s}_{m p}^{i}\right), \hat{\varepsilon}_{02}^{i}=\left(\mathbf{s}_{m n}^{i}, \mathbf{r}_{n}^{i} \times \mathbf{s}_{m n}^{i}\right), \hat{\varepsilon}_{\infty 1}^{i}=\left(\mathbf{0}, \mathbf{n}^{i}\right)$ and $\hat{\varepsilon}_{\infty 2}^{i}=\left(\mathbf{0}, \mathbf{s}_{m p}^{i} \times \mathbf{n}^{i}\right)$.

The (4S) ${ }^{i}$ linkage provides two independent translations and two independent rotations represented with the four independent twists $\hat{\varepsilon}_{01}^{i}, \hat{\varepsilon}_{02}^{i}, \hat{\varepsilon}_{\infty 1}^{i}$ and $\hat{\varepsilon}_{\infty 2}^{i}$ that span its twist system. Its twist graph represents the joints associated with the twists of a basis of its twist system. It is a virtual serial kinematic chain (Fig. 2b) composed of two virtual revolute joints $\mathrm{R}_{1}^{i}$ and $\mathrm{R}_{2}^{i}$ associated with $\hat{\varepsilon}_{01}^{i}$ and $\hat{\varepsilon}_{02}^{i}$ and two virtual

${ }^{1} \hat{\tau}$ and $\hat{\varepsilon}$ stand for unit wrench and unit twist, respectively

${ }^{2}$ By applying a linear transformation 
prismatic joints $\mathrm{P}_{1}^{i}$ and $\mathrm{P}_{2}^{i}$ associated with $\hat{\varepsilon}_{\infty 1}^{i}$ and $\hat{\varepsilon}_{\infty 2}^{i}$. These virtual joints are represented with dashed lines. Links 1, 2 and 3 are virtual links represented with dashed circles.

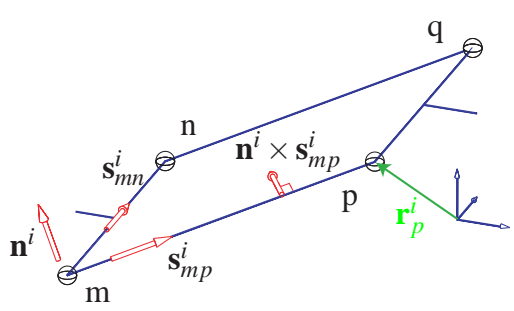

(a)

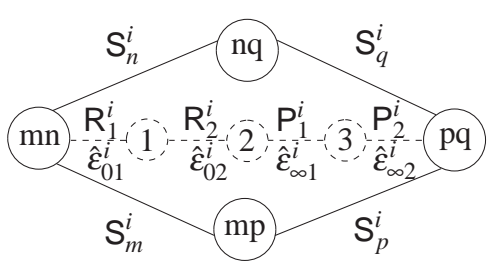

(b)

Fig. 2 (a) A (4S $)^{i}$ linkage; (b) Kinematic graph and twist graph of the $(4 S)^{i}$ linkage

\subsection{Constraint Wrenches of the H4 Robot}

Since the twist system of a $(4 \mathrm{~S})^{i}$ linkage is represented with a virtual chain composed of two revolute joints and two prismatic joints, a twist graph of the $H 4$ robot is obtained as shown in Fig. 3. The twist system $\mathrm{T}_{i}$ of the $i$ th $\operatorname{leg} l_{i}=\underline{\mathrm{R}}^{i}-(4 \mathrm{~S})^{i}$ (Fig. 4a) is equivalent to the twist system of a serial chain $\underline{\mathrm{R}}^{i}-\mathrm{R}_{1}^{i}-\mathrm{R}_{2}^{i}-\mathrm{P}_{1}^{i}-\mathrm{P}_{2}^{i}$ spanned by: $\hat{\varepsilon}_{01}^{i}=\left(\mathbf{s}_{1}^{i}, \mathbf{r}_{1}^{i} \times \mathbf{s}_{1}^{i}\right), \hat{\varepsilon}_{02}^{i}=\left(\mathbf{s}_{m n}^{i}, \mathbf{r}_{n}^{i} \times \mathbf{s}_{m n}^{i}\right), \hat{\varepsilon}_{03}^{i}=\left(\mathbf{s}_{m p}^{i}, \mathbf{r}_{p}^{i} \times \mathbf{s}_{m p}^{i}\right), \hat{\varepsilon}_{\infty 1}^{i}=\left(\mathbf{0}, \mathbf{n}^{i}\right)$ and $\hat{\varepsilon}_{\infty 2}^{i}=\left(\mathbf{0}, \mathbf{s}_{m p}^{i} \times \mathbf{n}^{i}\right)$. Note that $\mathbf{s}_{1}^{i}$ denotes a unit vector along the direction of $\underline{\mathrm{R}}^{i}$ joint axis. For the $i$ th leg $l_{i}, \mathbf{s}_{1}^{i}\left\|\mathbf{s}_{m n}^{i}\right\| \mathbf{s}_{p q}^{i}$ [17]. The constraint wrench system $\mathrm{W}_{i}$ of $l_{i}$ includes the wrenches that are reciprocal to all the twists in $\mathrm{T}_{i}$. Thus, the axis of a $\tau_{0}$ in $W_{i}$ is coplanar to the axes of $\hat{\varepsilon}_{01}^{i}, \hat{\varepsilon}_{02}^{i}$ and $\hat{\varepsilon}_{03}^{i}$ and orthogonal to the directions of $\hat{\varepsilon}_{\infty 1}^{i}$ and $\hat{\varepsilon}_{\infty 2}^{i}$. Therefore, $W_{i}$ does not contain any $\tau_{0}$. The direction of a $\tau_{\infty}$ in $W_{i}$ is orthogonal to $\mathbf{s}_{m n}^{i}, \mathbf{s}_{m p}^{i}$ and $\mathbf{s}_{1}^{i}$. It corresponds to the unit vector $\mathbf{s}_{m n}^{i} \times \mathbf{s}_{m p}^{i}=\mathbf{n}^{i}$. Hence, $\mathrm{W}_{i}$ is a 1 -system spanned by $\hat{\tau}_{\infty}^{i}=\left(\mathbf{0}, \mathbf{n}^{i}\right)$.

The chain $l_{12}$ being the connection of $l_{1}$ and $l_{2}$ in parallel, its wrench system $\mathrm{W}_{12}=\mathrm{W}_{1}+\mathrm{W}_{2}=\operatorname{span}\left(\hat{\tau}_{\infty}^{1}, \hat{\tau}_{\infty}^{2}\right)$. Thus, $l_{12}$ provides translations in any direction and cannot rotate about an axis directed along $\mathbf{n}^{1}$ or along $\mathbf{n}^{2}$. The direction of possible rotations is independent of these two vectors. This rotation is represented by the twist $\hat{\varepsilon}_{012}$ whose axis is directed along $\mathbf{s}_{m}^{12}=\mathbf{n}^{1} \times \mathbf{n}^{2}$. Therefore, $\mathrm{T}_{12}=\mathrm{W}_{12}^{\frac{1}{2}}=$ $\operatorname{span}\left(\hat{\varepsilon}_{\infty x}, \hat{\varepsilon}_{\infty x}, \hat{\varepsilon}_{\infty z}, \hat{\varepsilon}_{012}\right)$ where $\hat{\varepsilon}_{\infty x}, \hat{\varepsilon}_{\infty y}$ and $\hat{\varepsilon}_{\infty z}$ are the infinite pitch twists associated with translations along $\mathbf{x}, \mathbf{y}$ and $\mathbf{z}$, respectively. Similarly, the twist system of leg $l_{34}$ is $\mathrm{T}_{34}=\mathrm{W}_{34}^{\perp}=\operatorname{span}\left(\hat{\varepsilon}_{\infty x}, \hat{\varepsilon}_{\infty y}, \hat{\varepsilon}_{\infty \mathrm{z}}, \hat{\varepsilon}_{034}\right)$ and the axis of $\hat{\varepsilon}_{034}$ is directed along $\mathbf{s}_{m}^{34}=\mathbf{n}^{3} \times \mathbf{n}^{4}$.

The twist system of leg $L_{I}=l_{12}-\mathrm{R}_{I}$ is $\mathrm{T}_{I}=\mathrm{T}_{12}+\mathrm{T}_{R_{I}} . \mathrm{R}_{I}$ is the rotation about axis $Z_{I}$ (Fig. 4c) represented with the twist $\hat{\varepsilon}_{0 Z_{I}}=\left(\mathbf{z}, \mathbf{r}_{c} \times \mathbf{z}\right)$. Thus, $\mathrm{T}_{I}=$ $\operatorname{span}\left(\hat{\varepsilon}_{\infty x}, \hat{\varepsilon}_{\infty y}, \hat{\varepsilon}_{\infty z}, \hat{\varepsilon}_{012}, \hat{\varepsilon}_{0 Z_{I}}\right)$. Therefore, $\mathrm{W}_{I}=\mathrm{T}_{I}^{\perp}=\operatorname{span}\left(\hat{\tau}_{\infty I}\right)$ where $\hat{\tau}_{\infty I}=(\mathbf{0}, \mathbf{z} \times$ 


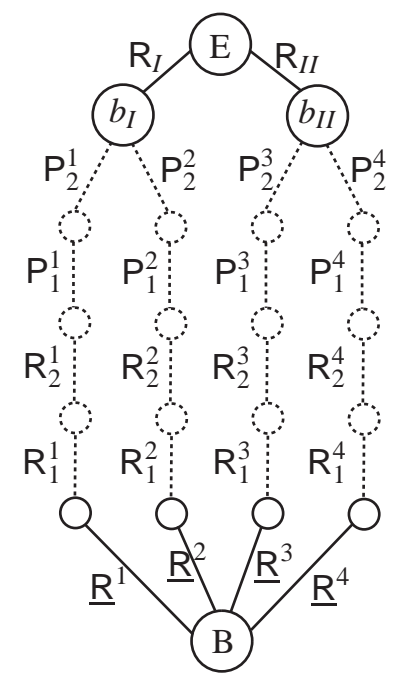

Fig. 3 Twist graph of the $H 4$ robot

$\left.\mathbf{s}_{m}^{12}\right)$. Likewise, $W_{I I}=\operatorname{span}\left(\hat{\tau}_{\infty I I}\right)$ where $\hat{\tau}_{\infty I I}=\left(\mathbf{0}, \mathbf{z} \times \mathbf{s}_{m}^{34}\right)$. Legs $L_{I}$ and $L_{I I}$ are mounted in parallel on the end effector of the $H 4$ robot. Thus, its constraint wrench system is:

$$
\mathrm{W}_{H 4}^{c}=\mathrm{W}_{I}+\mathrm{W}_{I I}=\operatorname{span}\left(\hat{\tau}_{\infty I}, \hat{\tau}_{\infty I I}\right)
$$

The end effector of the $H 4$ robot is constrained by two pure moments: $\hat{\tau}_{\infty I}=(\mathbf{0}, \mathbf{z} \times$ $\left.\mathbf{s}_{m}^{12}\right)$ and $\hat{\tau}_{\infty I I}=\left(\mathbf{0}, \mathbf{z} \times \mathbf{s}_{m}^{34}\right)$. It provides a Schönflies motion, i.e., three independent translations and one rotation about an axis of fixed direction along $\mathbf{z}$.

\subsection{Actuation Wrenches and Global Wrench System of the H4 Robot}

The actuated joints of the $H 4$ robot are the $\underline{\mathrm{R}}^{i}$ joints of legs $l_{i},(i=1, \ldots, 4)$. Legs $l_{1}$ and $l_{2}$ (respectively $l_{3}$ and $l_{4}$ ) are connected with $\mathrm{R}_{I}$ (respectively $\mathrm{R}_{I I}$ ). The actuation wrench system $\mathrm{W}_{1}^{a}$ of $l_{1}$ includes wrenches that are reciprocal to the twist of the $\mathrm{R}_{I}$ joint and all the twists in $\mathrm{T}_{1}$, except for the twist $\hat{\varepsilon}_{01}^{1}$ of $\underline{\mathrm{R}}^{1}$. Thus, $\mathrm{W}_{1}^{a}$ does not include any infinite pitch wrench. It is a 1 -system spanned by $\hat{\tau}_{0}^{1}$ of axis parallel to $\mathbf{s}_{m p}^{1}$, intersecting axis $Z_{I}$ and lying on the plane spanned by $m n$ and $m p$. The actuation wrench of $l_{1}$ is, thus, $\hat{\tau}_{0}^{1}=\left(\mathbf{s}_{u v}^{1}, \mathbf{r}_{v}^{1} \times \mathbf{s}_{u v}^{1}\right)$ with $\mathbf{s}_{u v}^{1} \| \mathbf{s}_{m p}^{1}$. Similarly, the actuation wrenches of legs $l_{2}, l_{3}$ and $l_{4}$ are $\hat{\tau}_{0}^{i}=\left(\mathbf{s}_{u v}^{i}, \mathbf{r}_{v}^{i} \times \mathbf{s}_{u v}^{i}\right),(i=2,3,4)$ with $\mathbf{s}_{u v}^{i} \| \mathbf{s}_{m p}^{i}$.

In a non-singular configuration, the four actuation wrenches $\hat{\tau}_{0}^{i}$ are independent and span the actuation wrench system of the $H 4$ robot: $\mathrm{W}_{H 4}^{a}=\operatorname{span}\left(\hat{\tau}_{0}^{i}\right)$, $(i=1, \ldots, 4)$. The global wrench system of the robot is: 


$$
\mathrm{W}_{H 4}=\mathrm{W}_{H 4}^{c}+\mathrm{W}_{H 4}^{a}=\operatorname{span}\left(\hat{\tau}_{\infty I}, \hat{\tau}_{\infty I I}, \hat{\tau}_{0}^{1}, \hat{\tau}_{0}^{2}, \hat{\tau}_{0}^{3}, \hat{\tau}_{0}^{4}\right)
$$

The legs of the $H 4$ robot apply two constraint moments and four actuation forces to its end effector. Its global wrench system is a 6-system. A parallel singularity occurs when the wrenches in the 6-system become linearly dependent and span a $k$-system with $k<6$.

\section{Singularity Analysis of the $H 4$ Robot}

\subsection{Wrench Diagram of the H4 Robot in $\mathbb{P}_{3}$}

A basis of the global wrench system $\mathrm{W}_{H 4}$ is composed of two constraint moments $\hat{\tau}_{\infty I}$ and $\hat{\tau}_{\infty I I}$, and four actuation forces $\hat{\tau}_{0}^{i}=\left(\mathbf{s}_{u v}^{i}, \mathbf{r}_{v}^{i} \times \mathbf{s}_{u v}^{i}\right),(i=1, \ldots, 4)$. Those wrenches are represented by two infinite lines and four finite lines in $\mathbb{P}_{3}$. To obtain the six extensors of the $H 4$ superbracket, we have to select twelve projective points on the six projective lines, i.e., two points on each one. The extensor of an infinite line is represented by two distinct infinite points. The extensor of a finite line can be represented by either two distinct finite points or one finite point and one infinite point, since any finite line has one point at infinity defined by its direction.

We know that $\hat{\tau}_{0}^{1}$ and $\hat{\tau}_{0}^{2}$ intersect axis $Z_{I}$. Likewise, $\hat{\tau}_{0}^{3}$ and $\hat{\tau}_{0}^{4}$ intersect axis $Z_{I I}$ (Fig. 4). Let a (respectively c) denote the intersection point of $\hat{\tau}_{0}^{1}$ (respectively $\hat{\tau}_{0}^{2}$ ) and $Z_{I}$ and let e (respectively g) denote the intersection point of $\hat{\tau}_{0}^{3}$ (respectively $\hat{\tau}_{0}^{4}$ ) and $Z_{I I}$. Besides, $Z_{I}$ and $Z_{I I}$ are parallel, i.e., ac and eg are parallel lines. They intersect in the infinite plane $\Pi_{\infty}$ at point $j=(\mathbf{z}, 0)$, which corresponds to the $\mathbf{z}$ direction. Note that an underlined letter stands for an infinite point. The finite line representing $\hat{\tau}_{0}^{1}=\left(\mathbf{s}_{u v}^{1}, \mathbf{r}_{v}^{1} \times \mathbf{s}_{u v}^{1}\right)$ can be defined by any two points on this line. Therefore, let $\hat{\tau}_{0}^{1}=\mathrm{a} \underline{\mathrm{b}}$ with $\underline{\mathrm{b}}=\left(\mathbf{s}_{u v}^{1}, 0\right)$ the infinite point of $\hat{\tau}_{0}^{1}$ defined by its direction. Likewise, $\hat{\tau}_{0}^{2}=\mathrm{c} \underline{\mathrm{d}}$ with $\underline{\mathrm{d}}=\left(\mathbf{s}_{u v}^{2}, 0\right), \hat{\tau}_{0}^{3}=\mathrm{e} \underline{\mathrm{f}}$ with $\underline{\mathrm{f}}=\left(\mathbf{s}_{u v}^{3}, 0\right)$ and $\hat{\tau}_{0}^{4}=\mathrm{g} \underline{\mathrm{h}}$ with $\underline{\mathrm{h}}=\left(\mathbf{s}_{u v}^{4}, 0\right)$.

The infinite line $\hat{\tau}_{\infty}^{i}=\left(\mathbf{0}, \mathbf{n}^{i}\right),(i=1, \ldots, 4)$ is the intersection with $\Pi_{\infty}$ of a finite plane $\Pi_{i}$ of normal $\mathbf{n}^{i}$. The intersection line of planes $\Pi_{1}$ and $\Pi_{2}$ is a finite line orthogonal to $\mathbf{n}^{1}$ and $\mathbf{n}^{2}$. The infinite point of this line is expressed as: $\underline{i}=\left(\mathbf{n}^{1} \times\right.$ $\left.\mathbf{n}^{2}, 0\right)=\left(\mathbf{s}_{m}^{12}, 0\right)$ and corresponds to the intersection point of $\hat{\tau}_{\infty}^{1}$ and $\hat{\tau}_{\infty}^{2}$. In the same vein, the intersection point of $\hat{\tau}_{\infty}^{3}$ and $\hat{\tau}_{\infty}^{4}$ is expressed as: $\underline{\mathbf{k}}=\left(\mathbf{n}^{3} \times \mathbf{n}^{4}, 0\right)=\left(\mathbf{s}_{m}^{34}, 0\right)$.

Let us consider the constraint moment $\hat{\tau}_{\infty I}=\left(\mathbf{0}, \mathbf{z} \times \mathbf{s}_{m}^{12}\right)$. Vector $\mathbf{z} \times \mathbf{s}_{m}^{12}$ is normal to any finite plane spanned by the two vectors $\mathbf{z}$ and $\mathbf{s}_{m}^{12}$. The infinite point of $\mathbf{s}_{m}^{12}$ is $\underline{\underline{i}}$ and the infinite point of $\mathbf{z}$ is $\underline{j}$. Therefore, $\hat{\tau}_{\infty I}=\underline{i j}$. Likewise, $\hat{\tau}_{\infty I I}=\underline{k j}$. We have selected the twelve points of the $H 4$ superbracket. The wrench diagram of the $H 4$ robot is represented in Fig. 4c. 


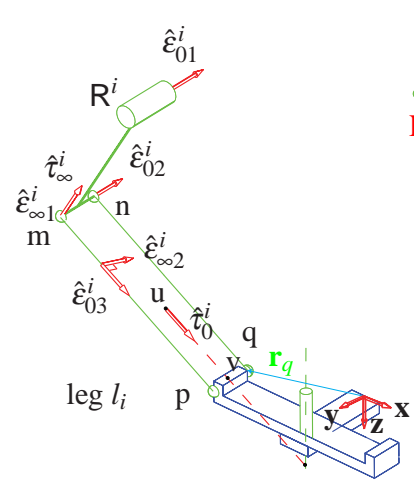

(a)

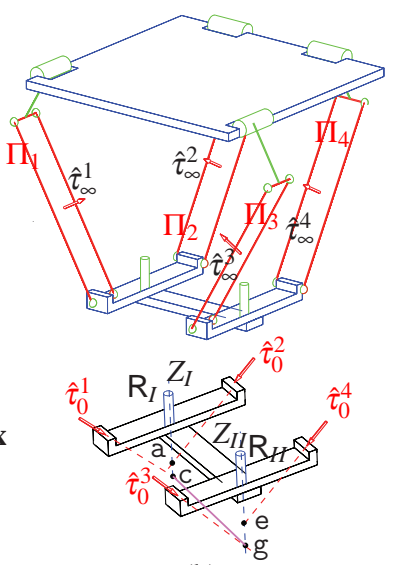

(b)

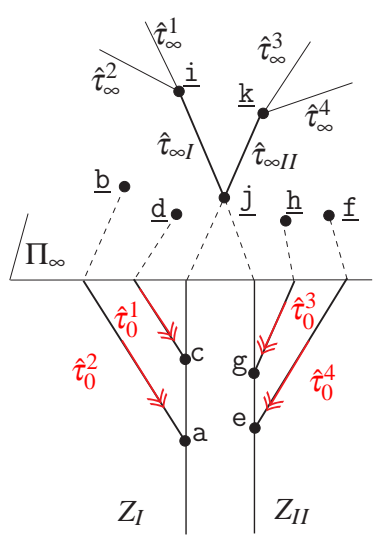

(c)

Fig. 4 (a) Twists of $\operatorname{leg} l_{i}$; (b) Constraint and actuation wrenches; (c) Wrench diagram in $\mathbb{P}_{3}$ of the H4 robot

\subsection{Superbracket of the H4 Robot}

The rows of the inverse Jacobian matrix of a parallel manipulator are Plücker coordinates of six lines in $\mathbb{P}_{3}$. The superjoin of these six vectors in $\mathbb{P}_{5}$ corresponds to the determinant of their six Plücker coordinate vectors up to a scalar multiple, which is the superbracket in GCA $\Lambda\left(V^{(2)}\right)$ [8]. Thus, a singularity occurs when these six Plücker coordinate vectors are dependent, which is equivalent to a superbracket equal to zero.

In [20], the theory of projective invariants has been used to decompose the superbracket into an expression having brackets involving 12 points selected on the axes of these lines. The expression of the $H 4$ superbracket is [ab $c \underline{d}$ e $\underline{f} g \underline{h} i j k j]$, it corresponds to twelve points selected in the robot wrench diagram. This expression can be developed into a linear combination (sum) of 24 bracket monomials [2, 20], each one being the product of three brackets of four projective points. The bracket of any four coplanar projective points is equal to zero. Hence, 19 amongst the 24 monomials are null. As detailed in [21], the simplified expression of this superbracket is:

$$
\begin{aligned}
& {[a \underline{b} c \underline{d} \underline{e} \underline{f} g \underline{h} \underline{i j} \underline{k j}]=[g \underline{i} \underline{k} \underline{j}]([a \underline{b} \underline{d} \underline{f}][c g \underline{h} \underline{j}]-[a \underline{b} \underline{d} \underline{h}][c g \underline{f} \underline{j}])} \\
& =[\mathrm{g} \underline{\mathrm{i}} \underline{\mathrm{k}} \underline{\mathrm{j}}][\mathrm{a} \underline{\mathrm{b}} \underline{\mathrm{d}} \underline{\dot{f}}][\mathrm{cg} \underline{\underline{\mathrm{h}}} \underline{\mathrm{j}}]
\end{aligned}
$$

where the dotted letters denote the permuted elements as mentioned in [8]. 


\subsection{Geometric Conditions for the H4 Robot Singularities}

From Eq. (5), a parallel singularity occurs when:

1. $[\mathrm{g} \underline{\underline{i}} \underline{\mathrm{k}} \underline{\mathrm{j}}]=0 \Leftrightarrow[\underline{\underline{i}} \underline{\mathrm{j}} \underline{\mathrm{j}}]=0 \Leftrightarrow \underline{\mathrm{i}}, \underline{\mathrm{j}}$ and $\underline{\mathrm{k}}$ belong to the same projective line. This condition is expressed in vector form as follows:

$$
\left(\mathbf{s}_{m}^{12} \times \mathbf{s}_{m}^{34}\right) \bullet \mathbf{z}=0
$$

where $\mathbf{s}_{m}^{12}=\mathbf{n}^{1} \times \mathbf{n}^{2}$ and $\mathbf{s}_{m}^{34}=\mathbf{n}^{3} \times \mathbf{n}^{4}$. This condition corresponds to the constraint singularities of the robot that occur when the legs lose their ability to constrain the motion of the end effector, which gains one or several DOFs. By solving Eq. (6), we obtain the different cases for this type of singularity as follows:

(a) $\mathbf{s}_{m}^{12} \times \mathbf{s}_{m}^{34}=0: \mathbf{s}_{m}^{12}$ and $\mathbf{s}_{m}^{34}$ are parallel, which happens when the intersection line of two amongst the four planes $\Pi_{i},(i=1, \ldots 4)$, is parallel to the intersection line of the two other planes. For example, when $\mathbf{n}^{1} \| \mathbf{n}^{3}$ and $\mathbf{n}^{2} \| \mathbf{n}^{4}$ as shown in Fig. 5a.

(b) $\mathbf{s}_{m}^{12} \| \mathbf{z}$, i.e., when the two planes $\Pi_{1}$ and $\Pi_{2}$ are vertical, their normal vectors $\mathbf{n}^{1}$ and $\mathbf{n}^{2}$ are in the horizontal plane and $\mathbf{s}_{m}^{12}=\mathbf{n}^{1} \times \mathbf{n}^{2}$ is parallel to $\mathbf{z}$. A similar case happens when $\mathbf{s}_{m}^{34} \| \mathbf{z}$;

(c) $\mathbf{n}^{1} \| \mathbf{n}^{2}$, i.e., the two planes $\Pi_{1}$ and $\Pi_{2}$ are parallel, and as a consequence $\mathbf{s}_{m}^{12}=0$. A similar case happens when $\mathbf{n}^{3} \| \mathbf{n}^{4}$

(d) $\mathbf{s}_{m}^{12}, \mathbf{s}_{m}^{34}$ and $\mathbf{z}$ are coplanar but not parallel to each other.

(e) $\mathbf{s}_{m}^{12}\left\|\mathbf{s}_{m}^{34}\right\| \mathbf{z}$, i.e., when the four planes $\Pi_{i},(i=1, \ldots 4)$, are vertical as shown in Fig. 5 b.

In cases (a), (b), (c) and (d), the two constraint moments $\hat{\tau}_{\infty}^{I}$ and $\hat{\tau}_{\infty}^{I I}$ are identical $\left(\mathbf{z} \times \mathbf{s}_{m}^{12} \| \mathbf{z} \times \mathbf{s}_{m}^{34}\right)$ or one of these moments is null. The constraint wrench system of the robot becomes a 1-system and its twist system a 5-system, and as a result, the manipulator gains one DOF. In case (e), the two constraints moments $\hat{\tau}_{\infty}^{I}$ and $\hat{\tau}_{\infty}^{I I}$ are null and the robot gains two DOFs.

2. [a $\underline{b} \underline{\mathrm{d}} \underline{\dot{f}}][\mathrm{cg} \underline{\underline{h}} \underline{j}]=0 \Leftrightarrow(\mathrm{a} \underline{\mathrm{b}} \underline{\mathrm{d}} \wedge \wedge \mathrm{cg} \underline{j}) \wedge \underline{\mathrm{f}} \underline{\mathrm{h}}=0 \Leftrightarrow$ the projective line $\underline{\mathrm{f}} \underline{\mathrm{h}}$ intersects with the intersection line of planes a $\underline{b} \underline{d}$ and $\operatorname{cg} \underline{j}$. This condition is expressed in vector form as follows:

$$
\left(\left[\mathbf{s}_{u v}^{1} \times \mathbf{s}_{u v}^{2}\right] \times\left[\left(\mathbf{r}_{g}-\mathbf{r}_{c}\right) \times \mathbf{z}\right]\right) \bullet\left(\mathbf{s}_{u v}^{3} \times \mathbf{s}_{u v}^{4}\right)=0
$$

This condition occurs when the legs cannot control the linear velocity of the end effector. The different cases for this condition can be established by solving Eq. (7). For example, when $\mathbf{s}_{u v}^{1} \| \mathbf{s}_{u v}^{2}$ and $\mathbf{s}_{u v}^{3} \| \mathbf{s}_{u v}^{4}$ (Fig. 5c). 


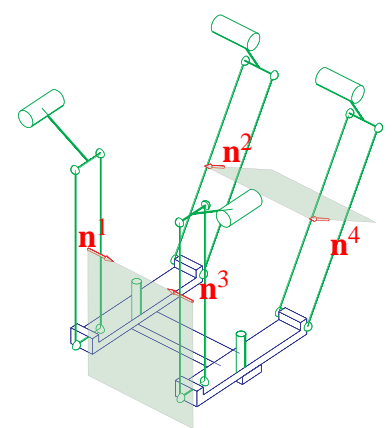

(a)

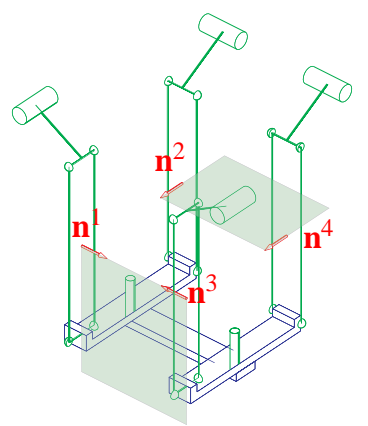

(b)

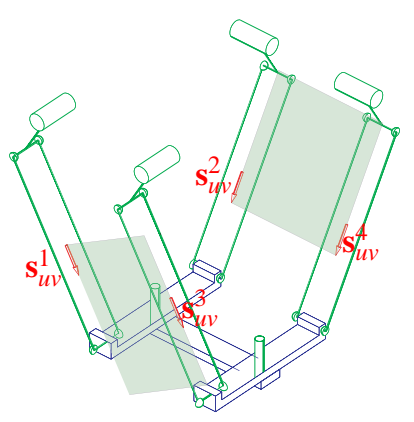

(c)

Fig. 5 Some singular configurations of the $H 4$ robot

\section{Conclusions}

In this paper, a general methodology was proposed to analyze the singularities of parallel manipulators with an articulated nacelle. The methodology consists of two main steps. First, the new concept of twist graph is used to simplify the constraint analysis. This graph is obtained with the theory of reciprocal screws. Then, a wrench diagram is obtained in order to derive a simplified expression of the superbracket decomposition. This expression is analyzed to provide geometric conditions for singular configurations. The methodology was explained through the singularity analysis of the $H 4$ robot. It can be also applied to analyze the singularities of other complex robots such as the Par4 ${ }^{3}$ [22].

\section{References}

1. J.P. Merlet. Singular Configurations of Parallel Manipulators and Grassmann Geometry. The International Journal of Robotics Research, 8: pp. 45-56, June 1989.

2. P. Ben-Horin and P. Shoham. Singularity Condition of Six-Degree-Of-Freedom Three-Legged Parallel Robots Based on Grassmann-Cayley Algebra. IEEE transactions on robotics, 22: pp. 577-590, 2006.

3. P. Ben-Horin and P. Shoham. Application of Grassmann-Cayley Algebra to Geometrical Interpretation of Parallel Robot Singularities. International Journal of Robotics Research, 1: pp. 127-141, 2009.

4. S.A. Joshi and L.W. Tsai. Jacobian Analysis of Limited-DOF Parallel Manipulators. Transactions of the ASME, 124, June 2002.

5. D. Kanaan, P. Wenger, S. Caro, and D. Chablat. Singularity Analysis of Lower-Mobility Parallel Manipulators Using Grassmann-Cayley Algebra. IEEE Transactions on Robotics, 25: pp. 995-1004, 2009.

6. H.B. Choi, Konno A., and Uchiyama M. Singularity Analysis of a Novel 4-DOFs Parallel Robot H4 by Using Screw Theory. Proceedings of DETC'03 ASME 2003 Design Engineering

\footnotetext{
${ }^{3}$ The commercial name of the Par4 is Quattro (http://www.adept.com/)
} 
Technical Conferences and Computers and Information in Engineering Conference, September 2-6 2003. Chicago, Illinois, USA.

7. J. Wu, Z. Yin, and Y. Xiong. Singularity Analysis of a Novel 4-dof Parallel Manipulator H4. The International Journal of Advanced Manufacturing Technology, 29: pp. 794-802, 2006.

8. N. L. White. Grassmann-Cayley Algebra and Robotics Applications. Handbook of Geometric Computing, Part VIII: pp. 629-656, 2005.

9. R. S. Ball. A Treatise On the Theory of Screws. Cambridge University Press, Cambridge, 1900.

10. K. J. Waldron. The Constraint Analysis of Mechanisms. Journal of Mechanisms, 1: pp. 101$114,1966$.

11. K. H. Hunt. Kinematic Geometry of Mechanisms. Clarendon Press, Oxford, 1978.

12. K. H. Hunt. Structural kinematics of in. parallel actuated robot arms. ASME J. of Mech. Transm. and Automn. in Design, 105: pp. 705-712, 1983

13. M. Mohamed and J. Duffy. A Direct Determination of the Instantaneous Kinematics of Fully Parallel Robot Manipulators. ASME J. of Mechanisms, Transmissions and Automation in Design, 107(2): pp. 226-229, 1985.

14. D. Zlatanov, I. Bonev, and C. Gosselin. Constraint Singularities of Parallel Mechanisms. IEEE ICRA'02, 1: pp. 496-502, 2002. Washington DC.

15. W. Kong and C.M. Gosselin. Type Synthesis of Parallel Mechanisms. Springer, August 2007.

16. T.S. Zhao, J.S. Dai, and Z. Huang. Geometric Analysis of Overconstrainted Parallel Manipulators with Three and Four Degrees of Freedom. JSME International Journal, 4(3): pp. 730-740, 2002.

17. F. Pierrot and O. Company. H4: A New Family of 4-dof Parallel Robots. Proc. of the IEEE / ASME Int. Conf. on Advanced Intelligent Mechatronics, Atlanta, Georgia, USA, pp. 508-513, September 1999.

18. F. Pierrot, F. Marquet, O Company, and T. Gil. H4 Parallel Robot: Modeling, Design and Preliminary Experiments. Proceedings of the IEEE International Conference on Robotics and Automation, pages 3256-3261, 2001.

19. G. Gogu. Structural Synthesis of Parallel Robots, volume 149 of Solid Mechanics and Its Applications. Springer, part 1: methodology edition, 2008.

20. T. McMillan and N.L. White The Dotted Straightening Algorithm Journal of Symbolic Computation, 11: pp. 471-482, 1991.

21. S. Amine, D. Kanaan, S. Caro, and P. Wenger. Singularity Analysis of The H4 Robot Using Grassmann-Cayley Algebra. Internal report, IRCCyN 2010, http://www.irccyn.ecnantes.fr/ caro/Publications/ARK2010/IntReport.pdf

22. V. Nabat, M. de la O Rodriguez, O. Company, S. Krut, and F. Pierrot. Par4: Very High Speed Parallel Robot for Pick-and-Place. Intelligent Robots and Systems, IEEE/RSJ International Conference, pp. 553-558, August 2005. 